\title{
Utility and costs of radiologist interpretation of perioperative imaging in patients with traumatic single-level thoracolumbar fractures
}

\author{
Michael H. Weber, MD, PhD, ${ }^{1}$ Lojan Sivakumaran, MD, ${ }^{1}$ Maryse Fortin, PhD, ${ }^{1}$ Alisson R. Teles, MD, ${ }^{2}$ \\ Jeff D. Golan, MD, ${ }^{2}$ Carlo Santaguida, MD, ${ }^{2}$ Peter Jarzem, MD, ${ }^{1}$ and Thierry Pauyo, MD ${ }^{1}$ \\ Departments of ${ }^{1}$ Orthopedics and ${ }^{2}$ Neurosurgery, McGill University Health Centre, Montreal, Quebec, Canada
}

OBJECTIVE The cost of spine management is rising. As diagnostic imaging accounts for approximately $10 \%$ of total patient care spending, there is interest in determining if economies could be made with regard to the routine consultation of radiology for image interpretation. In the context of spine trauma, both the spine surgeon and the radiologist interpret perioperative imaging. Authors of the present study investigated the impact of radiologist interpretation of perioperative imaging from patients with traumatic single-level thoracolumbar fractures given that spine surgeons are expected to be comfortable interpreting pathologies of the musculoskeletal system.

METHODS The authors conducted a retrospective review of all patients presenting with a single-level thoracolumbar fracture treated at the McGill University Health Centre in the period from January 2003 to December 2010. The time between image capture and radiologist interpretation as well as the number of extraskeletal and/or incidental findings was extracted from the radiology reports on all perioperative images including radiographic, fluoroscopic, and CT images. The cost of interpretation was obtained from the provincial health insurance entity of Quebec.

RESULTS Eighty-two patients met the study inclusion criteria. Radiologists took a median of 1 day (IQR 0-5.5 days) to interpret preoperative radiographs. Intraoperative fluoroscopic images and postoperative radiographs were read by the radiologist a median of 19 days (IQR 4-56.75 days) and 34 days (IQR 1-137.5 days) after capture, respectively ( $\mathrm{p}<$ 0.05). Preoperative radiologist dictations reported extraskeletal and/or incidental findings for $8.1 \%$ of radiographs; there were no intraoperative or postoperative extraskeletal findings beyond those previously reported on the preoperative radiographs. Radiologists took a median of 1 day (IQR $0-1$ day) to read both preoperative and postoperative CT scans; extraskeletal and/or incidental findings were present in $46.2 \%$ of preoperative reports and $4.5 \%$ of postoperative reports. There were no intraoperative or postoperative radiological findings that provoked reoperation. A total of 66 intraoperative fluoroscopy images and 225 postoperative radiographs were read for a cost of $\$ 1399.20$ and $\$ 1867.50$ (Canadian dollars), respectively, for radiologist interpretation. This cost amounted to $40.3 \%$ of all perioperative image interpretation spending.

CONCLUSIONS In the management of single-level thoracolumbar fractures, radiologists add information to the diagnostic picture when interpreting preoperative radiographs and perioperative CT scans; however, the interpretation of intraoperative fluoroscopic images and postoperative radiographs comes with significant delay, does not add additional information, and represents an area of potential cost and professional-resource reduction.

https://thejns.org/doi/abs/10.3171/2017.4.SPINE16923

KEY WORDS thoracolumbar; spine; trauma; radiologist; image; interpretation

$\mathrm{T}$ OTAL spending associated with the management of spine-related disorders is on the rise. ${ }^{6}$ Whether equivalent outcomes can be achieved while reducing costs remains unclear. One key area of interest lies in the management of spine trauma patients. Hospitalization and operative costs comprise the majority of spending for these patients; ${ }^{8}$ however, another important area to consider is the reliance (or overreliance) on consultation services. In the context of the increasing use of imaging in modern health care $^{2}$ and the often automatic referral of hospital- 
based imaging to radiology, the true need for dual interpretation of perioperative imaging by spine surgeons and radiologists has yet to be investigated.

In the current paradigm, both spine surgeons and radiologists perform independent reads of spine images, including radiographs and CT scans. Spine surgeons are expected to be competent in interpreting the musculoskeletal aspect of patients' imaging; however, they generally rely on radiologists to detect extraskeletal findings. Thus, in a preoperative patient, the two specialists have complementary roles. However, in the intraoperative and postoperative periods - where imaging may have stable extraskeletal findings and be subject to long delays for interpretationthe role of dual interpretation remains unclear.

The purpose of the current study was to investigate, in the context of single-level thoracolumbar fractures, the costs and benefits associated with the dual interpretation of all perioperative imaging by both orthopedic surgeons and radiologists. The time to interpretation and extraskeletal findings by the radiologist were examined to determine whether the readings had an impact on patient care. The cost of the radiologist readings was also analyzed to determine the extent of possible cost reductions if dual reads were not performed, when appropriate.

\section{Methods}

We conducted a retrospective review of patients treated for acute traumatic single-level thoracolumbar fractures at the McGill University Health Centre (MUHC) between January 2003 and December 2010. Only patients who had received acute surgical management were included in the study. Patients who had fractures associated with hardware failure, as a result of an operative complication, or attributable to osteoporosis were excluded. Patients whose imaging had been ordered from an outside institution or lacked final interpretation by the radiologist by the end of the study period were also excluded. As an institutional protocol, all patients with spinal instrumentation undergo postoperative radiography before discharge. In the scenario of thoracolumbar fractures, postoperative CT scanning is done at the discretion of the surgical team to assess hardware position and decompression when necessary.

Patient demographics including age, sex, and level of vertebral fractures were recorded. Perioperative imagespreoperative and postoperative radiographs, intraoperative fluoroscopic images, and preoperative and postoperative CT scans-and their dictated radiology reports were retrieved using the MUHC InteleViewer picture archiving and communication system (PACS) software. The dictations were considered final when the attending radiologist had signed them electronically.

The time between the date of image capture and the date of final interpretation was recorded. For preoperative images, the time to interpretation was compared with the date of operation to help determine whether the interpretation was involved in patient management. Extraskeletal findings - defined as structural abnormalities to any solid or hollow organs or other region of interest confined to the thoracic or abdominal cavity - and incidental findings were retrieved from these reports. The cost associated with the
TABLE 1. Summary of data on 82 patients with traumatic single-level thoracolumbar fractures

\begin{tabular}{lc}
\hline \multicolumn{1}{c}{ Category } & Value \\
\hline No. of patients & \\
\hline Male & $55(67 \%)$ \\
\hline Female & $27(33 \%)$ \\
\hline Average patient age in years & \\
\hline Male & 40.1 \\
\hline Female & 39.4 \\
\hline Overall (range) & $39.9(18-81)$ \\
\hline Location of fracture & \\
\hline L1-5 & $61(74.4 \%)$ \\
\hline T5-12 & $21(25.6 \%)$ \\
\hline
\end{tabular}

radiologist interpretation of each image was obtained from the Registry of Health Insurance of Quebec (RAMQ), a universal single-payer government-administrated system.

This study was approved by the Health and Research Ethics Board of the MUHC. The median value and interquartile range were reported, with ANOVA statistically significant at $\mathrm{p}<0.05$. Statistical analysis was performed using Microsoft Excel (Microsoft Corp.).

\section{Results}

Eighty-two patients met the criteria for study inclusion. The mean patient age was 39.9 years (range 18-81 years). Men made up $67 \%$ of the patients. Additional patient characteristics are listed in Table 1.

\section{Preoperative and Postoperative Radiographs and Intraoperative Fluoroscopy: Time to Interpretation}

Two hundred seventy-six radiographs were obtained in the perioperative period. Forty patients $(48.8 \%)$ had preoperative radiographs taken as part of their initial evaluation by the emergency department. Radiologists took a median of 1 day (IQR 0-5.5 days) to read and interpret the preoperative radiographs. The majority of the patients $(83.4 \%)$ with preoperative radiographs had a final interpretation on the day of surgery.

Sixty-six surgically treated patients had intraoperative fluoroscopy images available. Radiologists took a median of 19 days (IQR 4-56.75 days) to interpret these images.

All surgically treated patients had postoperative radiographs taken. Radiologists took a median of 34 days (IQR 1-137.5 days) to interpret the postoperative radiographs. Approximately one-third of the postoperative radiographs (36.4\%) were read within a week of being taken.

There was a significant difference in the median number of days it took the radiologist to read the preoperative and postoperative radiographs and intraoperative fluoroscopic images $(\mathrm{p}<0.001$; Fig. 1$)$.

\section{Preoperative and Postoperative CT Time to Interpretation}

A total of $91 \mathrm{CTs}$ were taken as part of the perioperative surgical care. Sixty-five patients $(79.3 \%)$ had preoperative 


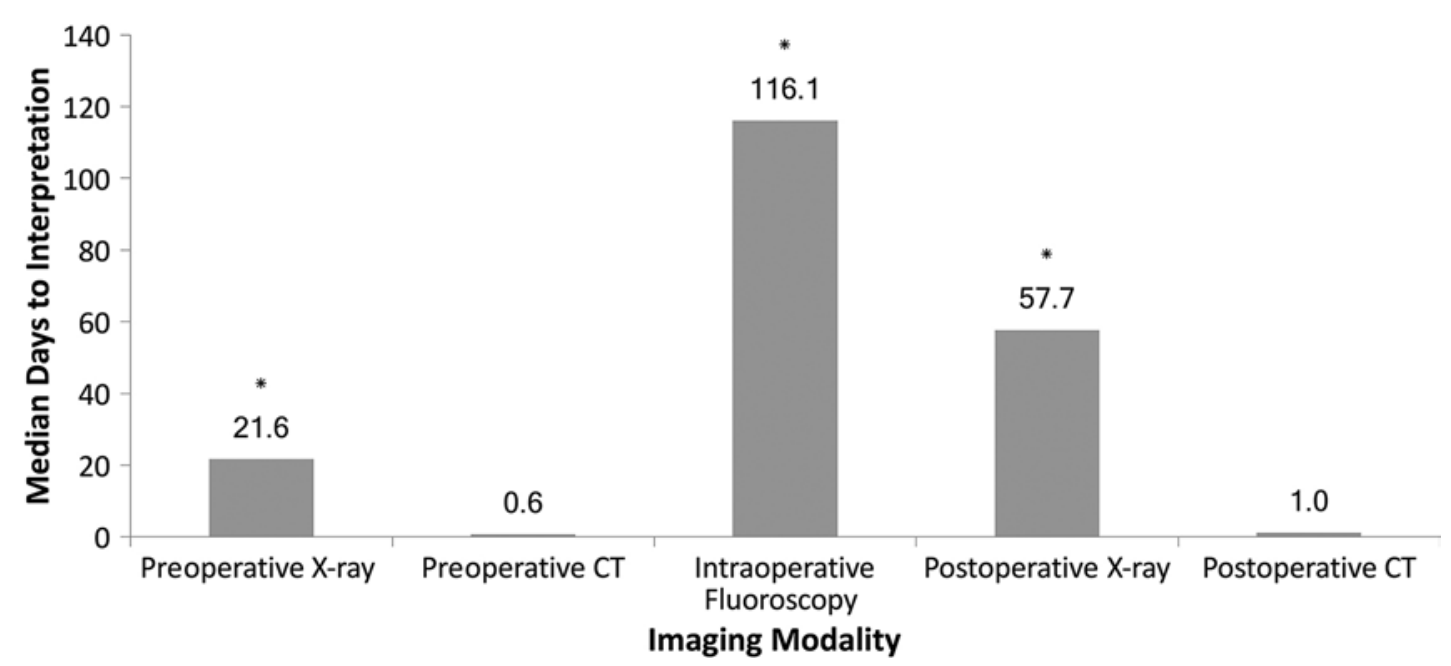

FIG. 1. Graph showing the median number of days to radiologist interpretation of perioperative images for patients with single-level thoracolumbar fracture. ${ }^{*} p<0.05$, ANOVA.

CTs taken as part of their initial evaluation. The median number of days that radiologists took to read and interpret the preoperative CT was less than 1 day (IQR $0-1$ day). All patients with preoperative CTs had a final radiologist interpretation on the day of surgery.

A total of $23.2 \%$ of patients had postoperative CTs, which took a median of 1 day (IQR $0-1$ day) to be interpreted by the radiologist. There was no significant difference in the median number of days it took the radiologists to read the preoperative and postoperative CTs $(\mathrm{p}>0.05$; Fig. 1).

\section{Extraskeletal and/or Incidental Findings and Clinical Management}

There were 35 extraskeletal and/or incidental findings identified by radiologists on all perioperative imaging.
These findings included solid organ lesions, pulmonary nodules, and paravertebral hematomas. There was a significant difference in the incidence of extraskeletal and/ or incidental findings on preoperative $(8.1 \%)$, intraoperative $(0.0 \%)$, and postoperative radiographs $(0.0 \% ; \mathrm{p}<0.01)$. There was also a significant difference in the incidence of extraskeletal and/or incidental findings on preoperative (46.2\%) and postoperative (4.5\%) CTs (p <0.01; Fig. 2).

There were no findings in the radiologist reports on the intraoperative and postoperative imaging (including radiograph, fluoroscopic image, and CT) that provoked reintervention by the surgical team.

\section{Cost}

The total health care cost engendered by perioperative imaging interpretation was $\$ 8103.50$ (all values expressed

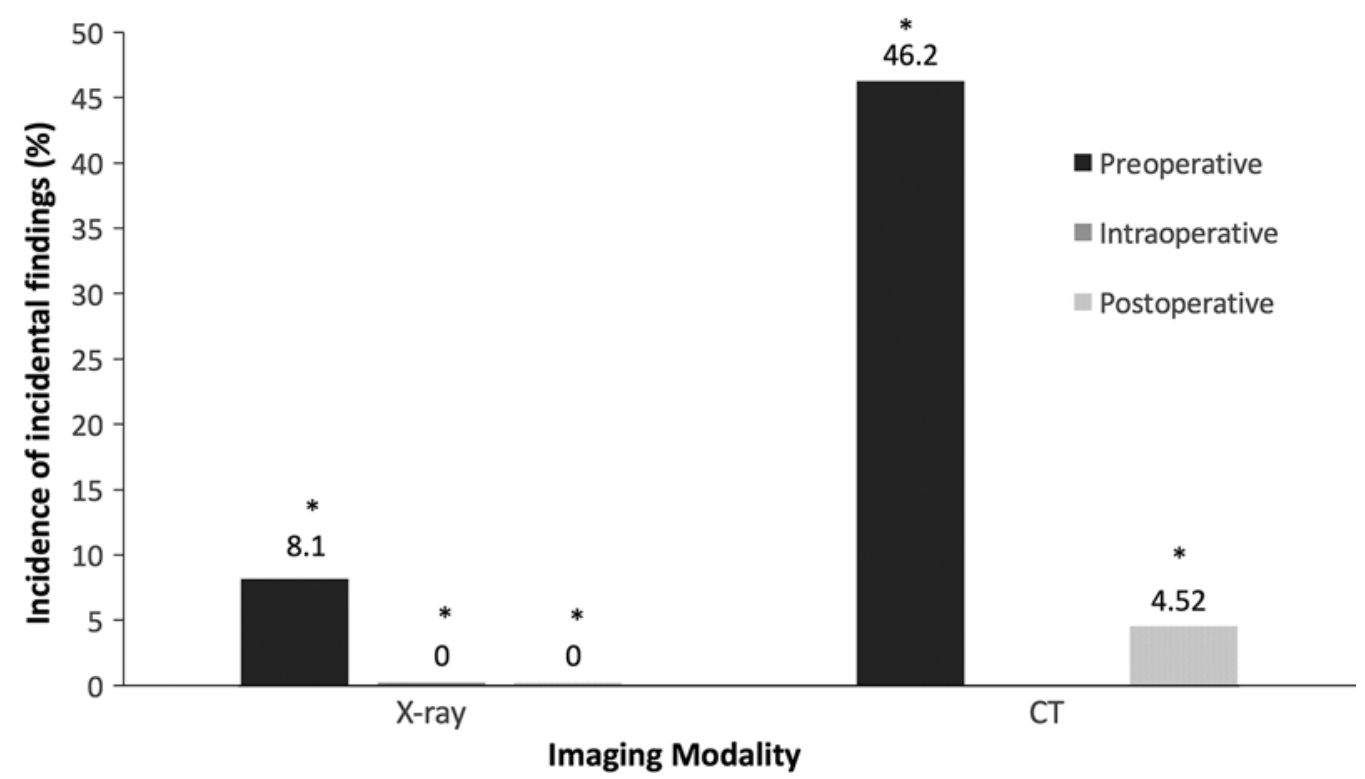

FIG. 2. Incidence of extraskeletal and/or incidental findings identified by radiologists on perioperative radiographs and CT in patients with single-level thoracolumbar fractures. "Intraoperative" refers to intraoperative fluoroscopy. " $p<0.05$, ANOVA. 
TABLE 2. Costs associated with the interpretation of perioperative imaging for single-level thoracolumbar fractures by radiologists

\begin{tabular}{lrcc}
\hline Imaging & $\begin{array}{c}\text { No. of } \\
\text { Images }\end{array}$ & $\begin{array}{c}\text { Cost of } \\
\text { Interpretation }\end{array}$ & $\begin{array}{c}\text { Cost per } \\
\text { 1000 Patients }\end{array}$ \\
\hline Preop & & & \\
\hline Radiograph & 51 & 423.30 & $5,162.20$ \\
\hline CT & 69 & 3346.50 & $40,810.98$ \\
\hline Total & 120 & 3769.80 & $45,973.17$ \\
\hline Intraop & & & \\
\hline Fluoroscopy & 66 & 1399.20 & $17,063.41$ \\
\hline Postop & & & \\
\hline Radiograph & 225 & 1867.50 & $22,774.39$ \\
\hline CT & 22 & 1067.00 & $13,012.20$ \\
\hline Total & 247 & 2934.50 & $35,786.59$ \\
\hline
\end{tabular}

Costs expressed in Canadian dollars. Radiologists reimbursed by RAMQ in MUHC between 2003 and 2010.

in Canadian dollars) for the 82 patients (Table 2). A total of $\$ 423.30$ was spent for the interpretation of preoperative radiographs, whereas $\$ 1399.20$ and $\$ 1867.50$ were spent for interpretation of intraoperative fluoroscopy and postoperative radiographs, respectively. The cost associated with radiologist interpretation of preoperative and postoperative CTs was $\$ 3346.50$ and $\$ 1067.00$, respectively. The cost associated with radiologist assessment of intraoperative fluoroscopy and postoperative radiographs represented $40.3 \%$ of the total cost of all perioperative radiological interpretations.

\section{Discussion}

Diagnostic imaging accounts for $10 \%$ of total health care costs, and the amount of imaging performed in North America is on the rise. ${ }^{2,4}$ Results of the present study suggest that while radiologists provide additional information to the diagnostic picture when interpreting preoperative radiographs as well as preoperative and postoperative CTs in the context of spine trauma management, their consultation for intraoperative fluoroscopic images and postoperative radiographs may not add additional information to the diagnostic picture and is associated with a high cost.

In the preoperative period, the radiologists provided timely interpretations of radiographs and reported a significant number of extraskeletal findings. Taken together, these observations indicate that the radiologists' interpretations were available for spine management and that their findings were beyond the scope of the spine surgeon's expertise. The latter point is especially important as previous studies have reported a high correlation between thoracolumbar fractures and other thoracic or abdominal injuries.., 9

However, the value of having radiologists assess intraoperative fluoroscopy and postoperative radiographs is less clear. Their interpretations did not yield any additional extraskeletal findings and were subject to long delays after image capture. The previously established equivalence of radiologists and orthopedic surgeons in interpreting trau- matic orthopedic fractures on radiography ${ }^{3,7}$ seems to indicate that having these images interpreted only by spine surgeons would not affect patient care. The finding that no intraoperative or postoperative imaging provoked reintervention supports this conclusion. A case example is provided in Fig. 3. Previously published studies have indeed found that dual interpretation of radiographs in a similar setting-although not spine fractures-would not alter patient care. ${ }^{1,3}$

As regards $\mathrm{CT}$, both preoperative and postoperative scans were read in a timely manner and both had extraskeletal findings. There were significantly more extraskeletal findings in the preoperative versus postoperative reads, probably because the field of scan was smaller. Firstly, this indicates that the preoperative read by the radiologist has utility. Secondly, although the radiologist noted fewer extraskeletal findings on postoperative CTs than on preoperative CTs, they still found some; given the potential implication of any incidental finding on imaging, the role of the radiologist cannot be underestimated in interpreting postoperative CT.

In interpreting the study results conservatively, it appears that the routine consultation of radiologists for interpreting intraoperative fluoroscopy and postoperative radiographs in single-level thoracolumbar fracture patients should be scrutinized given the lack of extraskeletal findings and the delay to interpretation. However, our results also reaffirm the utility of radiologists in interpreting preoperative radiographs and perioperative $\mathrm{CT}$ using the minimal criteria of extraskeletal and/or incidental findings and time to interpretation. Based on the RAMQ insurance system, the elimination of intraoperative fluoroscopic and postoperative radiographic reads by radiologists would represent a cost savings opportunity of $40 \%$ on overall reads in spine trauma patients, which in turn represents more than $\$ 39,000$ per 1000 patients. For a medical community in which both patient outcomes and cost containment are crucial, this study provides insight into a specific area in which economies could be made.

\section{Study Limitations}

The study population size was modest. One could argue that the potential cost savings identified in our study (\$39,000 per 1000 patients) by eliminating radiology interpretation of intraoperative fluoroscopy and postoperative radiographic imaging could be minimal in comparison with the medicolegal costs of a single missed diagnosis in 1000 patients. On the other hand, our study included consecutive single-level thoracolumbar fractures over an 8 -year period in a trauma center, and we were not able to identify a single radiological interpretation of intraoperative fluoroscopic and postoperative radiographic images that impacted the management of these patients. We believe that the results of this study should encourage the performance of a larger multicenter study to investigate the need for mandatory radiology interpretation of postoperative spine imaging in this scenario.

In this study, we used the presence of extraskeletal findings and the time to interpretation as surrogates for the utility of radiologist interpretations; the next step would be to examine the impact of these interpretations on pa- 

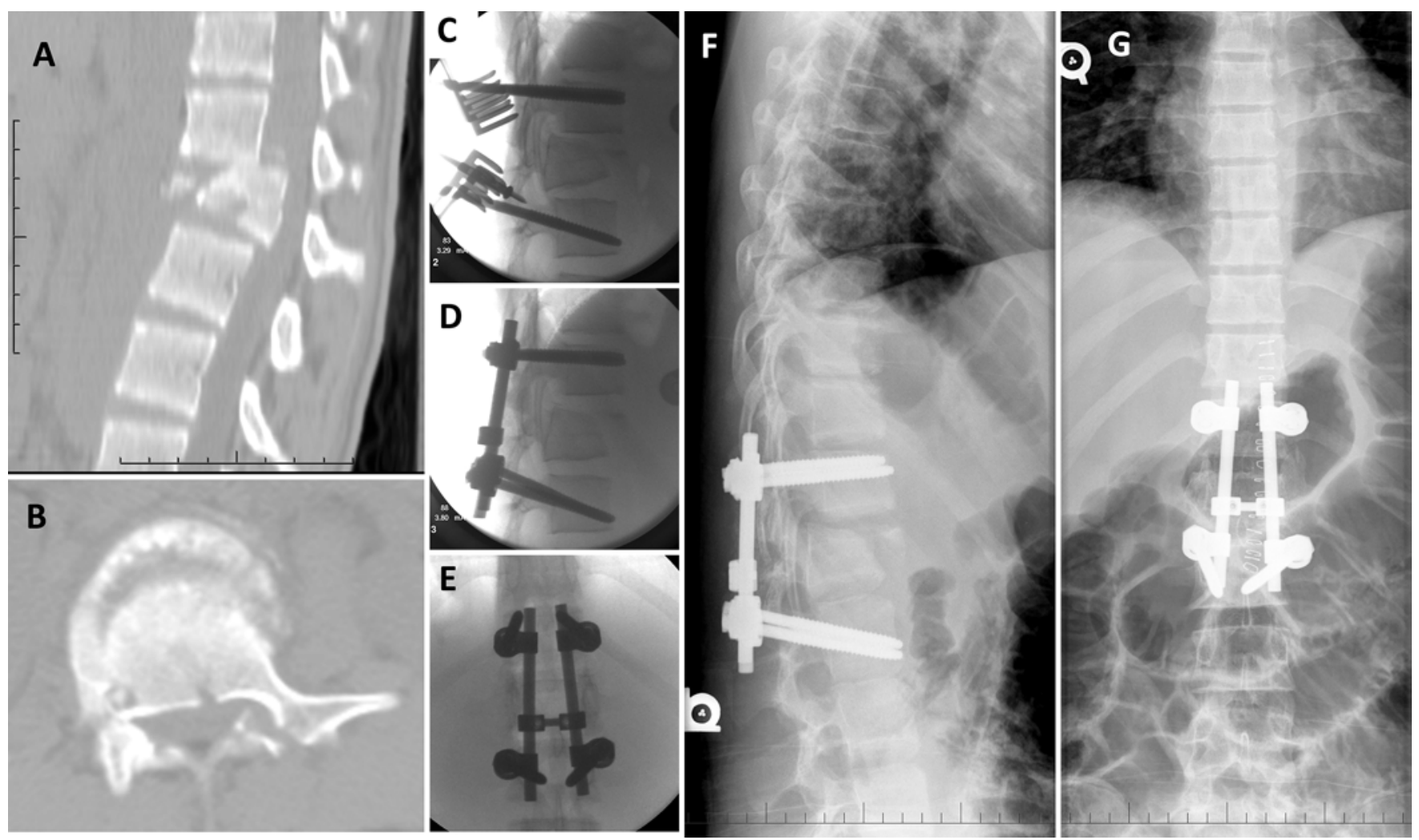

FIG. 3. Images obtained in a 19-year-old male victim of a motor vehicle accident who had presented with no neurological deficits. Preoperative CT scans (A and B) were interpreted by the radiologist on the same day as patient admission and surgery and revealed "an unstable L1 burst fracture with a significant retropulsion." Intraoperative fluoroscopy images (C-E) were interpreted 11 days after surgery as "intraoperative images of spinal fusion." Postoperative radiographs (F and $\mathbf{G})$ were interpreted by the radiologist 12 days after surgery as "postoperative control of T12 to L2 posterior spinal fixation." As for the surgeon's interpretation, the fluoroscopic images revealed good reduction of the fracture and adequate hardware position. The postoperative radiographs demonstrated normal thoracolumbar alignment.

tient outcomes. This would have particular impact in the context of a prospective trial. The patient records were not verified for interpretation of the imaging by the orthopedic surgeon; however, the fact that none of the intraoperative and postoperative radiographic findings by the radiologist provoked surgical reintervention (or had extraskeletal findings) indicates that patient management probably would not have changed based on radiologist interpretations of these images.

This study was not designed to assess the appropriateness of ordering postoperative imaging in the setting of single-level thoracolumbar fractures. Generally, all patients who undergo spinal instrumentation at our institution undergo postoperative radiography. A CT scan is usually obtained in selected cases to assess canal decompression and hardware position. In this sense, savings would be even greater by eliminating unnecessary postoperative imaging. A larger prospective study is necessary to evaluate the appropriateness of routine postoperative images in this scenario.

Finally, this study is only representative of one center's experience in managing such patients. We defined the time for interpretation as the final sign-off by the attending radiology staff, not considering the time for preliminary dictations done by trainees. Thus, the delay to inter- pretation of the imaging studies represents the reality at our institution and may not reflect the experience at other centers. However, regardless of the time to interpretation, our results demonstrated that the dual interpretation of intraoperative fluoroscopy and postoperative radiography did not impact the management of patients with singlelevel thoracolumbar fractures.

\section{Conclusions}

The present study provides insight into the true need for routine consultation of radiology for the assessment of imaging in the perioperative period for the spine trauma patient. While radiologists reported a number of extraskeletal and/or incidental findings on preoperative radiography and pre- and postoperative CT in a timely fashion, their reads of intraoperative fluoroscopic images and postoperative radiographs added little additional information. Furthermore, these latter interpretations tended to be delayed and represented a large proportion of the image-interpretation costs. Thus, it appears that the routine consultation of radiology for the interpretation of intraoperative fluoroscopic images and postoperative radiographs represents an area of potential cost and professional service economy in patient management. Future studies should be 
directed toward the clinical outcomes of patients who do not have routine radiological consultation for their image interpretation.

\section{References}

1. Anglen J, Marberry K, Gehrke J: The clinical utility of duplicate readings for musculoskeletal radiographs. Orthopedics 20:1015-1019, 1997

2. Beinfeld MT, Gazelle GS: Diagnostic imaging costs: are they driving up the costs of hospital care? Radiology 235:934939, 2005

3. Bosse MJ, Brumback RJ, Hash C: Medical cost containment: analysis of dual orthopedic/radiology interpretation of X-rays in the trauma patient. J Trauma 38:220-222, 1995

4. Canadian Institute for Health Information: Medical Imaging in Canada 2012. Ottawa: Canadian Institute for Health Information, 2013 (https://www.cihi.ca/en/mit_ summary_2012_en.pdf) [Accessed May 11, 2017]

5. Reid AB, Letts RM, Black GB: Pediatric chance fractures: association with intra-abdominal injuries and seatbelt use. J Trauma 30:384-391, 1990

6. Rihn JA, Currier BL, Phillips FM, Glassman SD, Albert TJ: Defining the value of spine care. J Am Acad Orthop Surg 21:419-426, 2013

7. Turen CH, Mark JB, Bozman R: Comparative analysis of radiographic interpretation of orthopedic films: is there redundancy? J Trauma 39:720-721, 1995

8. van der Roer N, de Bruyne MC, Bakker FC, van Tulder MW,
Boers M: Direct medical costs of traumatic thoracolumbar spine fractures. Acta Orthop 76:662-666, 2005

9. Xia T, Tian JW, Dong SH, Wang L, Zhao QH: Non-spinalassociated injuries with lumbar transverse process fractures: influence of segments, amount, and concomitant vertebral fractures. J Trauma Acute Care Surg 74:1108-1111, 2013

\section{Disclosures}

The authors report no conflict of interest concerning the materials or methods used in this study or the findings specified in this paper.

\section{Author Contributions}

Conception and design: Weber, Pauyo. Acquisition of data: Sivakumaran, Golan, Jarzem, Pauyo. Analysis and interpretation of data: Weber, Sivakumaran, Pauyo. Drafting the article: Weber, Sivakumaran, Pauyo. Critically revising the article: Weber, Fortin, Teles, Golan, Santaguida, Jarzem. Reviewed submitted version of manuscript: Teles. Statistical analysis: Fortin. Administrative/ technical/material support: Fortin, Santaguida. Study supervision: Weber.

\section{Correspondence}

Michael H. Weber, Department of Orthopedics, McGill University Health Centre, 1650 Cedar Ave., Montreal, QC H3G 1A4, Canada. email: michael.weber@hotmail.com. 